\title{
Spatial Form of a Hamiltonian Dysthe Equation for Deep-Water Gravity Waves
}

\author{
Philippe Guyenne ${ }^{1, *} \oplus$, Adilbek Kairzhan ${ }^{2}$, Catherine Sulem ${ }^{2}$ and Boyang $\mathrm{Xu}^{1}$ \\ 1 Department of Mathematical Sciences, University of Delaware, Newark, DE 19716, USA; boyangxu@udel.edu \\ 2 Department of Mathematics, University of Toronto, Toronto, ON M5S 2E4, Canada; \\ kairzhan@math.toronto.edu (A.K.); sulem@math.utoronto.ca (C.S.) \\ * Correspondence: guyenne@udel.edu
}

check for updates

Citation: Guyenne, P.; Kairzhan, A.; Sulem, C.; Xu, B. Spatial Form of a Hamiltonian Dysthe Equation for Deep-Water Gravity Waves. Fluids 2021, 6, 103. https://doi.org/ $10.3390 /$ fluids 6030103

Academic Editor: Alexander I. Dyachenko

Received: 31 January 2021

Accepted: 22 February 2021

Published: 3 March 2021

Publisher's Note: MDPI stays neutral with regard to jurisdictional claims in published maps and institutional affiliations.

Copyright: (c) 2021 by the authors. Licensee MDPI, Basel, Switzerland. This article is an open access article distributed under the terms and conditions of the Creative Commons Attribution (CC BY) license (https:// creativecommons.org/licenses/by/ $4.0 /)$.
Abstract: An overview of a Hamiltonian framework for the description of nonlinear modulation of surface water waves is presented. The main result is the derivation of a Hamiltonian version of Dysthe's equation for two-dimensional gravity waves on deep water. The reduced problem is obtained via a Birkhoff normal form transformation which not only helps eliminate all non-resonant cubic terms but also yields a non-perturbative procedure for surface reconstruction. The free surface is reconstructed from the wave envelope by solving an inviscid Burgers' equation with an initial condition given by the modulational Ansatz. Particular attention is paid to the spatial form of this model, which is simulated numerically and tested against laboratory experiments on periodic groups and short-wave packets. Satisfactory agreement is found in all these cases.

Keywords: deep-water gravity waves; Dysthe equation; Hamiltonian systems; modulation theory; numerical simulations

\section{Introduction}

Because the cubic nonlinear Schrödinger (NLS) equation for water waves had been shown to have a limited range of applicability, Dysthe [1] extended this model to the next order in the perturbation analysis. The resulting equation is now widely used in the water wave community because of its efficiency and ability to describe realistic waves, in particular with moderately large steepnesses. Among the new features are effects from the wave-induced mean flow which lead to improvement in the stability properties of finite-amplitude waves. Due to its higher-order nonlinear terms, Dysthe's equation is also able to capture the asymmetric development of propagating wave packets. Originally derived in the context of gravity waves on deep water, it has since been extended to various other settings and has produced a large literature: e.g., finite depth [2], gravitycapillary waves [3], exact linear dispersion [4], dissipation [5], higher order [6], just to cite a few references. Interest in Dysthe's equation lies in the fact that it is more amenable to mathematical analysis and numerical simulation than the full water wave equations, while being able to capture salient features of weakly nonlinear wave packets. In particular, the associated computational cost is significantly less compared to that from direct (fully nonlinear) numerical solvers [7-10].

In this asymptotic regime, solutions are sought as mild modulations of monochromatic waves. Besides the wave steepness, another small parameter is associated with the spectral width relative to the dominant carrier wavenumber (the so-called narrowband approximation), and accordingly evolution equations are written for the complex wave envelope. Such models have typically been derived via the method of multiple scales from the full water wave equations $[1,2,5]$, or as a reduction of the Fourier integro-differential equation that was first proposed by Zakharov [11] and later refined by Krasitskii [12] for resonantly interacting water waves in the small-amplitude limit $[3,4,13]$. These studies provide a formal derivation and analysis of Dysthe's equation, and it is only recently that 
rigorous results on its well-posedness have been obtained [14,15]. The rigorous justification of envelope equations for water waves has so far been proved for the NLS equation only $[16,17]$. This mathematical question remains an open problem for Dysthe's equation.

Despite these advances, early versions of Dysthe's equation share a fundamental shortcoming: unlike the NLS Equation [18], they are not Hamiltonian partial differential equations whereas the full water wave equations are. From a modeling point of view, it is desirable that a reduced model inherits important properties of the original system. Regardless of whether the modulational analysis is carried out from the full water wave equations or from Zakharov's equation, an envelope model is not guaranteed to be Hamiltonian unless there is a way to ensure that a symplectic structure is retained together with some conserved energy.

Progress in this direction has recently been made by Gramstad and Trulsen [19] and Craig et al. [20] who proposed Hamiltonian versions of Dysthe's equation. Gramstad and Trulsen [19] used Krasitskii's refined version of Zakharov's equation as a basis for their modulational analysis of three-dimensional gravity waves on arbitrary depth. Craig et al. [20] considered the two-dimensional problem of gravity waves on deep water and combined results from [21-25] to derive such models. Their formal approach involves a reduction of the basic Hamiltonian to Birkhoff normal form at fourth order, followed by a series of canonical transformations together with a procedure of homogenization. In particular, the normal form transformation helps eliminate all non-resonant cubic terms, which leads to a simple and elegant expression for the reduced Hamiltonian. These calculations are facilitated by the fact that the Dirichlet-Neumann operator has an explicit Taylor expansion, as devised by Craig and Sulem [7].

Another important step in the solution process for such approximations is the reconstruction of the free surface from the wave envelope, which is performed at a postprocessing stage but yet affects the predictions' accuracy. In classical (i.e., non-Hamiltonian) derivations of Dysthe's equation as well as in the alternate derivation by Gramstad and Trulsen [19], this reconstruction is carried out perturbatively in terms of a Stokes' expansion based on the Ansatz for the solutions' form. Contributions are included up to an order that is consistent with the order of approximation for Dysthe's equation. By contrast, Craig et al. [20] proposed a non-perturbative approach for surface reconstruction which requires solving a nonlinear partial differential equation (PDE) associated with the Birkhoff normal form transformation. This PDE for the surface elevation takes the generic form of an inviscid Burgers' equation. Higher-order harmonics of the surface wave spectrum are automatically generated from the carrier component through nonlinear interactions according to this PDE. As a consequence, the entire solution process in this approach fits within a Hamiltonian framework, meaning that both the envelope equation and the nonlinear PDE for surface reconstruction possess a well-defined Hamiltonian structure. Preliminary tests in [20] show very good agreement between this approximation and numerical simulations of the full water wave equations for the temporal evolution of Benjamin-Feir instability of Stokes waves on deep water.

Furthermore, Craig et al. [20] provided an alternate spatial form of their Hamiltonian model in which the role of the spatial and temporal variables is interchanged. Spatial forms of Dysthe's equation have also received much attention in the literature as they are more suitable for comparison with time series recorded by fixed probes along the wave tank in laboratory experiments, or recorded by an array of buoys in field experiments [26-28]. Such a manipulation of the independent variables is applied to the envelope equation as well as to the procedure for surface reconstruction, which here requires a nontrivial adjustment of Burgers' equation associated with the Birkhoff normal form transformation.

In the present paper, after giving an overview of this Hamiltonian approach and reviewing recent results by Craig et al. [20], we propose an improved strategy for the surface computation. Focusing on the spatial dynamics (with the spatial and temporal variables switched), we further introduce a dimensionless rescaled form of the equations 
to facilitate comparison with laboratory experiments. We perform numerical simulations and test them under various wave conditions.

On a related note, we also point out recent work by Dyachenko et al. [29-31] on a special reduction of Zakharov's equation for two-dimensional gravity waves on deep water (which has been referred to as the compact or super compact equation). This reduction is made possible in this case by taking advantage of explicit analytical expressions of 4 -wave resonances and by identifying exact cancellation of terms. The resulting simpler form is similar to that for Dysthe's equation (while being free of the narrowband approximation) and still possesses a Hamiltonian structure. It is thus better suited than Zakharov's equation for analysis and computation, and numerical results have indicated that it is able to describe large-amplitude rogue waves and even waves reaching near the breaking point. A spatial Hamiltonian version of this compact equation can also be derived, as shown in [29,32].

The remainder of this paper is organized as follows. Section 2 introduces the basic mathematical formulation for two-dimensional gravity waves on deep water, including the Hamiltonian form of the governing equations and the Birkhoff normal form transformation to eliminate all non-resonant cubic terms. Section 3 describes the main steps in our Hamiltonian perturbation approach, including the modulational Ansatz to specify the special form of solutions in this asymptotic regime, the resulting Hamiltonian equation for temporal dynamics of the wave envelope, and the corresponding procedure for surface reconstruction. Section 4 presents the spatial version of this approximation together with its dimensionless rescaled form. Numerical simulations of our spatial model are then shown in Section 5 where it is compared to the classical formulation. Tests against laboratory experiments on periodic groups and short-wave packets are also presented, for which satisfactory agreement is obtained. Finally, concluding remarks are given in Section 6.

\section{Governing Equations}

2.1. Hamiltonian Formulation

Consider a two-dimensional fluid of infinite depth

$$
S(\eta)=\{x \in \mathbb{R},-\infty<y<\eta(x, t)\},
$$

bounded above by a free surface $\{y=\eta(x, t)\}$. The variables $(x, y)$ denote the horizontal and vertical coordinates respectively, and the variable $t$ denotes time. For an incompressible, inviscid and irrotational flow, the velocity potential $\varphi(x, y, t)$ satisfies the boundary value problem

$$
\begin{aligned}
& \nabla^{2} \varphi=0 \quad \text { in } S(\eta), \\
& \partial_{t} \eta+\left(\partial_{x} \eta\right)\left(\partial_{x} \varphi\right)-\partial_{y} \varphi=0 \quad \text { at } y=\eta(x, t), \\
& \partial_{t} \varphi+\frac{1}{2}|\nabla \varphi|^{2}+g \eta=0 \text { at } y=\eta(x, t), \\
& \partial_{y} \varphi \rightarrow 0 \text { as } y \rightarrow-\infty,
\end{aligned}
$$

where $g$ is the acceleration due to gravity and $\nabla=\left(\partial_{x}, \partial_{y}\right)^{\top}$ is the spatial gradient.

The Laplace problem (1)-(4) can be reduced from one posed inside the entire fluid domain to one posed on the free surface alone. As proposed by Craig and Sulem [7], a key ingredient is to introduce the Dirichlet-Neumann operator (DNO)

$$
G(\eta) \xi=\left.\left(-\partial_{x} \eta, 1\right)^{\top} \cdot \nabla \varphi\right|_{y=\eta}
$$


which takes Dirichlet data $\xi(x, t)=\varphi(x, \eta(x, t), t)$ at the free surface, solves Laplace's Equation (1) for $\varphi$ with boundary condition (4), and returns the corresponding Neumann data (i.e., the surface normal velocity). Equations (1)-(4) then reduce to

$$
\begin{aligned}
& \partial_{t} \eta=G(\eta) \xi \\
& \partial_{t} \xi=-g \eta-\frac{1}{2\left(1+\left(\partial_{x} \eta\right)^{2}\right)}\left[\left(\partial_{x} \xi\right)^{2}-(G(\eta) \xi)^{2}-2\left(\partial_{x} \eta\right)\left(\partial_{x} \xi\right) G(\eta) \xi\right],
\end{aligned}
$$

which constitute a closed Hamiltonian system for the two conjugate variables $\eta$ and $\xi$ in Zakharov's formulation of the water wave problem [7,11]. These can be cast into canonical form

$$
\partial_{t}\left(\begin{array}{l}
\eta \\
\xi
\end{array}\right)=J\left(\begin{array}{c}
\delta_{\eta} H \\
\delta_{\xi} H
\end{array}\right)=\left(\begin{array}{cc}
0 & 1 \\
-1 & 0
\end{array}\right)\left(\begin{array}{c}
\delta_{\eta} H \\
\delta_{\xi} H
\end{array}\right),
$$

where the $2 \times 2$ matrix $J$ represents the symplectic structure of the system, and the Hamiltonian

$$
H(\eta, \xi)=\frac{1}{2} \int\left[\xi G(\eta) \xi+g \eta^{2}\right] \mathrm{d} x
$$

coincides with the total energy and is an invariant for the temporal dynamics. All integrals are evaluated over $\mathbb{R}$ but this notation is omitted for convenience.

Due to analyticity properties for a sufficiently regular free surface [33], the DNO can be written via a convergent Taylor series expansion

$$
G(\eta)=\sum_{j=0}^{\infty} G_{j}(\eta),
$$

about the quiescent state $\eta=0$. Each term $G_{j}$ is homogeneous of degree $j$ in $\eta$ and is determined recursively [7]. The first three terms in this expansion are

$$
\begin{aligned}
G_{0} & =|D|, \\
G_{1}(\eta) & =D \eta D-G_{0} \eta G_{0}, \\
G_{2}(\eta) & =-\frac{1}{2}\left(|D|^{2} \eta^{2} G_{0}+G_{0} \eta^{2}|D|^{2}-2 G_{0} \eta G_{0} \eta G_{0}\right),
\end{aligned}
$$

where $D=-i \partial_{x}$ (so that its Fourier symbol is $k$ ). The reader is referred to $[9,21,24,34-36]$ for other applications of this formulation to long-wave perturbation calculations as well as direct numerical simulations of nonlinear wave phenomena.

With this observation, the Hamiltonian can be decomposed as

$$
H=H_{2}+H_{3}+H_{4}+\ldots
$$

where

$$
\begin{gathered}
H_{2}(\eta, \xi)=\frac{1}{2} \int\left(\xi G_{0} \xi+g \eta^{2}\right) \mathrm{d} x \\
\begin{aligned}
H_{3}(\eta, \xi)=\frac{1}{2} \int \xi G_{1}(\eta) \xi \mathrm{d} x= & \frac{1}{2} \int \xi\left(D \eta D-G_{0} \eta G_{0}\right) \xi \mathrm{d} x \\
& =-\frac{1}{2} \int \eta\left[(D \xi)^{2}+\left(G_{0} \xi\right)^{2}\right] \mathrm{d} x \\
H_{4}(\eta, \xi)=\frac{1}{2} \int \xi G_{2}(\eta) \xi \mathrm{d} x= & -\frac{1}{4} \int \xi\left(|D|^{2} \eta^{2} G_{0}+G_{0} \eta^{2}|D|^{2}-2 G_{0} \eta G_{0} \eta G_{0}\right) \xi \mathrm{d} x \\
= & -\frac{1}{2} \int \eta\left[\eta\left(G_{0} \xi\right)\left(D^{2} \xi\right)-\left(G_{0} \xi\right) G_{0}\left(\eta G_{0} \xi\right)\right] \mathrm{d} x
\end{aligned}
\end{gathered}
$$


represent the quadratic, cubic and quartic contributions respectively, in terms of $(\eta, \xi)$. The first contribution $\mathrm{H}_{2}$ describes the linear behavior while the next contributions $\mathrm{H}_{3}$ and $\mathrm{H}_{4}$ describe nonlinear mechanisms of 3-wave and 4-wave interactions, respectively.

Our Hamiltonian approach amounts to applying a sequence of canonical transformations to the Hamiltonian (9) of the system, and to its symplectic structure (8). As a consequence, a Hamiltonian model is obtained at each level of approximation.

\subsection{Normal Form Transformations}

Because 3-wave resonances are not allowed for deep-water gravity waves, the Hamiltonian $H(\eta, \xi)$ can be simplified by eliminating all non-essential cubic terms through a suitable canonical transformation [12]. This idea is inherent to the general theory of normal form transformations for Hamiltonian systems and has been successfully applied to the water wave problem, starting with the seminal work of Zakharov [11]. Birkhoff normal form up to a given order $m$ results from a sequence of canonical changes of variables, so that the Taylor expansion of the transformed Hamiltonian up to order $m$ contains only essential (i.e., resonant) terms.

In the two-dimensional problem of gravity waves on infinite depth [25], the normal form transformation for $m=3$ can be defined as the flow at $s=-1$ subject to the vector field

$$
\partial_{s}\left(\begin{array}{l}
\eta \\
\xi
\end{array}\right)=\left(\begin{array}{cc}
0 & 1 \\
-1 & 0
\end{array}\right)\left(\begin{array}{l}
\delta_{\eta} K_{3} \\
\delta_{\xi} K_{3}
\end{array}\right)
$$

for some auxiliary Hamiltonian $K_{3}$, and with initial condition at $s=0$ being tied to the original physical variables $(\eta, \xi)$. The variable $s$ plays the role of an evolutionary variable for this auxiliary Hamiltonian system. More specifically, the normal form is defined through a Taylor expansion of the Hamiltonian about $s=0$,

$$
\begin{aligned}
\left.H(\eta, \xi)\right|_{s=-1}= & \left.H(\eta, \xi)\right|_{s=0}-\left.\frac{d H}{d s}(\eta, \xi)\right|_{s=0}+\left.\frac{1}{2} \frac{d^{2} H}{d s^{2}}(\eta, \xi)\right|_{s=0}+\ldots, \\
= & \left.H(\eta, \xi)\right|_{s=0}-\left.\left\{K_{3}, H\right\}(\eta, \xi)\right|_{s=0}+\left.\frac{1}{2}\left\{K_{3},\left\{K_{3}, H\right\}\right\}(\eta, \xi)\right|_{s=0}+\ldots, \\
= & \left.H_{2}(\eta, \xi)\right|_{s=0}+\left.H_{3}(\eta, \xi)\right|_{s=0}+\left.H_{4}(\eta, \xi)\right|_{s=0}-\left.\left\{K_{3}, H_{2}\right\}(\eta, \xi)\right|_{s=0} \\
& -\left.\left\{K_{3}, H_{3}\right\}(\eta, \xi)\right|_{s=0}+\left.\frac{1}{2}\left\{K_{3},\left\{K_{3}, H_{2}\right\}\right\}(\eta, \xi)\right|_{s=0}+\ldots
\end{aligned}
$$

where $K_{3}$ is homogeneous of degree $3, H_{n}$ is homogeneous of degree $n$ and the Poisson bracket

$$
\left\{K_{3}, H_{n}\right\}=\int\left[\left(\delta_{\eta} H_{n}\right)\left(\delta_{\xi} K_{3}\right)-\left(\delta_{\xi} H_{n}\right)\left(\delta_{\eta} K_{3}\right)\right] \mathrm{d} x,
$$

is of degree $n+1$. All cubic terms are eliminated if

$$
\left.H_{3}(\eta, \xi)\right|_{s=0}-\left.\left\{K_{3}, H_{2}\right\}(\eta, \xi)\right|_{s=0}=0,
$$

which is satisfied for

$$
K_{3}(\eta, \xi)=\frac{1}{2} \int(-i \operatorname{sgn}(D) \eta)^{2}|D| \xi \mathrm{d} x .
$$

Derivatives in the above expansion are related to Poisson brackets through

$$
\frac{d H}{d s}=\int\left[\left(\delta_{\eta} H\right)\left(\partial_{s} \eta\right)+\left(\delta_{\xi} H\right)\left(\partial_{s} \xi\right)\right] \mathrm{d} x=\left\{K_{3}, H\right\},
$$

by virtue of (11). The operator $-i \operatorname{sgn}(D)$ is the Hilbert transform in a Fourier multiplier form. Accordingly, it is convenient to introduce

$$
\left(\begin{array}{l}
\widetilde{\eta} \\
\widetilde{\xi}
\end{array}\right)=P_{0}\left(\begin{array}{l}
\eta \\
\xi
\end{array}\right)=\left(\begin{array}{cc}
-\mathrm{i} \operatorname{sgn}(D) & 0 \\
0 & -\mathrm{i} \operatorname{sgn}(D)
\end{array}\right)\left(\begin{array}{l}
\eta \\
\xi
\end{array}\right),
$$


so that

$$
K_{3}(\widetilde{\eta}, \widetilde{\xi})=\frac{1}{2} \int \widetilde{\eta}^{2} \partial_{x} \widetilde{\zeta} \mathrm{d} x
$$

and

$$
\partial_{s}\left(\begin{array}{l}
\widetilde{\eta} \\
\widetilde{\zeta}
\end{array}\right)=J_{0}\left(\begin{array}{l}
\delta_{\widetilde{\eta}} K_{3} \\
\delta_{\widetilde{\xi}} K_{3}
\end{array}\right)=\left(\begin{array}{cc}
0 & -1 \\
1 & 0
\end{array}\right)\left(\begin{array}{l}
\delta_{\widetilde{\eta}} K_{3} \\
\delta_{\widetilde{\xi}} K_{3}
\end{array}\right) .
$$

The corresponding symplectic structure is given by $J_{0}=P_{0} J P_{0}^{\top}=-J$. This auxiliary system produces two equations: one for $\widetilde{\eta}$,

$$
\partial_{s} \widetilde{\eta}-\widetilde{\eta} \partial_{x} \widetilde{\eta}=0,
$$

is an inviscid Burgers' equation, while the other for $\widetilde{\xi}$,

$$
\partial_{s} \widetilde{\xi}-\widetilde{\eta} \partial_{x} \widetilde{\zeta}=0,
$$

is its linearization along Burgers' flow. The new Hamiltonian then reads

$$
\begin{aligned}
H & =H_{2}(\eta, \xi)+H_{4}(\eta, \xi)-\left\{K_{3}, H_{3}\right\}(\eta, \xi)+\frac{1}{2}\left\{K_{3},\left\{K_{3}, H_{2}\right\}\right\}(\eta, \xi)+R_{5}, \\
& =H_{2}(\eta, \xi)+H_{4}(\eta, \xi)-\frac{1}{2}\left\{K_{3}, H_{3}\right\}(\eta, \xi)+R_{5}, \\
& =H_{2}(\eta, \xi)+H_{4}(\eta, \xi)-H_{4}(-\mathrm{i} \operatorname{sgn}(D) \eta, \xi)+R_{5},
\end{aligned}
$$

where the leading nonlinear contributions are now quartic terms, and follows from (12) together with the identity

$$
\frac{1}{2}\left\{K_{3}, H_{3}\right\}(\eta, \xi)=\frac{1}{2} \int \xi G_{2}(-\mathrm{i} \operatorname{sgn}(D) \eta) \xi \mathrm{d} x=H_{4}(-\mathrm{i} \operatorname{sgn}(D) \eta, \xi),
$$

as shown in [25]. By construction, this normal form transformation is canonical and thus preserves the symplectic structure $J$ in (8). More details on Birkhoff normal forms for the water wave problem can be found in $[11,25,37,38]$.

\section{Hamiltonian Dysthe's Equation}

\subsection{Modulational Ansatz}

Here we describe the special form of solutions to be sought in this asymptotic regime. We first make the change to complex symplectic coordinates $z(x, t)$ and $\bar{z}(x, t)$ as defined by

$$
\left(\begin{array}{l}
z \\
\bar{z}
\end{array}\right)=P_{1}\left(\begin{array}{l}
\eta \\
\xi
\end{array}\right)=\frac{1}{\sqrt{2}}\left(\begin{array}{cc}
a(D) & \mathrm{i} a^{-1}(D) \\
a(D) & -\mathrm{i} a^{-1}(D)
\end{array}\right)\left(\begin{array}{l}
\eta \\
\xi
\end{array}\right)
$$

where the symbol ? stands for complex conjugation, and

$$
a(D)=\sqrt[4]{\frac{g}{G_{0}}} .
$$

System (8) then becomes

$$
\partial_{t}\left(\begin{array}{l}
z \\
\bar{z}
\end{array}\right)=J_{1}\left(\begin{array}{c}
\delta_{z} H \\
\delta_{\bar{z}} H
\end{array}\right)=\left(\begin{array}{cc}
0 & -\mathrm{i} \\
\mathrm{i} & 0
\end{array}\right)\left(\begin{array}{c}
\delta_{z} H \\
\delta_{\bar{z}} H
\end{array}\right),
$$

after adjusting the symplectic structure $J_{1}=P_{1} J P_{1}^{\top}$.

The next calculation involves the modulational Ansatz

$$
\left(\begin{array}{l}
u \\
\bar{u}
\end{array}\right)=P_{2}\left(\begin{array}{l}
z \\
\bar{z}
\end{array}\right)=\varepsilon^{-1}\left(\begin{array}{cc}
e^{-\mathrm{i} k_{0} x} & 0 \\
0 & e^{\mathrm{i} k_{0} x}
\end{array}\right)\left(\begin{array}{l}
z \\
\bar{z}
\end{array}\right),
$$


which implies that we are seeking solutions in the form of near-monochromatic waves with carrier wavenumber $k_{0}>0$ and with slowly varying complex envelope $u(X, t)$ depending on $X=\varepsilon x$. The dimensionless parameter $\varepsilon=k_{0} A_{0} \ll 1$ is a measure of the wave steepness ( $A_{0}$ denotes a characteristic wave amplitude for the free surface). It is also a measure of the wave spectrum's narrowness around $k=k_{0}$. The resulting equations of motion are

$$
\partial_{t}\left(\begin{array}{l}
u \\
\bar{u}
\end{array}\right)=J_{2}\left(\begin{array}{c}
\delta_{u} H \\
\delta_{\bar{u}} H
\end{array}\right)=\varepsilon^{-1}\left(\begin{array}{cc}
0 & -\mathrm{i} \\
\mathrm{i} & 0
\end{array}\right)\left(\begin{array}{c}
\delta_{u} H \\
\delta_{\bar{u}} H
\end{array}\right),
$$

where $J_{2}=\varepsilon P_{2} J_{1} P_{2}^{\top}$. The additional factor $\varepsilon$ in this definition of $J_{2}$ reflects the change in symplectic structure from the spatial rescaling $x \rightarrow X=\varepsilon x$ and plays a part in defining the relevant slow time scale for the resulting systems. More details on these canonical transformations in the context of Hamiltonian models for interfacial flows can be found in $[22,24,39]$.

\subsection{Envelope Equation for Temporal Dynamics}

Naturally, the Hamiltonian (16) is also modified through the changes of variables (17) and (19). The first transformation (17) performs a diagonalization of the quadratic part

$$
H_{2}=\int \bar{z} \omega(D) z \mathrm{~d} x,
$$

in terms of normal modes $(z, \bar{z})$ and the linear dispersion relation

$$
\omega(D)=\left(g G_{0}\right)^{1 / 2}=(g|D|)^{1 / 2},
$$

for deep-water gravity waves. The second transformation (19) sets the stage for the expansion of $H$ in powers of $\varepsilon$. These calculations are facilitated by the fact that the DNO admits a Taylor expansion with an explicit form for its Taylor terms.

Due to the multiscale nature of this problem (fast oscillations in $x$ and slow modulation in $X)$, it is important to understand the action of Fourier multiplier operators on multiscale functions. The following result from [40] describes this action.

Theorem 1. Let $\alpha(D)$ be a Fourier multiplier. For sufficiently smooth functions $f(X)$, we have

$$
\begin{aligned}
\alpha(D)\left(e^{\mathrm{i} k_{0} x} f(X)\right) & =e^{\mathrm{i} k_{0} x} \alpha\left(k_{0}+\varepsilon D_{X}\right) f(X), \\
& =e^{\mathrm{i} k_{0} x}\left(\sum_{j=0}^{n} \frac{\varepsilon^{j}}{j !} \partial_{k}^{j} \alpha\left(k_{0}\right) D_{X}^{j} f(X)+O\left(\varepsilon^{n+1}\right)\right) .
\end{aligned}
$$

As an example,

$$
\begin{aligned}
G_{0}\left(e^{\mathrm{i} k_{0} x} f(X)\right) & =e^{\mathrm{i} k_{0} x}\left|k_{0}+\varepsilon D_{X}\right| f(X), \\
& =e^{\mathrm{i} k_{0} x}\left(k_{0}^{2}+2 \varepsilon k_{0} D_{X}+\varepsilon^{2} D_{X}^{2}\right)^{1 / 2} f(X), \\
& =e^{\mathrm{i} k_{0} x}\left(k_{0}+\varepsilon D_{X}+\ldots\right) f(X) .
\end{aligned}
$$

Furthermore, the presence of multiple scales needs to be appropriately dealt with in order to extract the nontrivial dynamics of the slowly varying wave envelope. The scale separation lemma of Craig et al. [21,23] is used to address this homogenization problem for our Hamiltonian system. As a result, terms with fast oscillations essentially homogenize to zero and thus do not contribute to the effective Hamiltonian, which implies that only 4 -wave resonances (i.e., terms with the same number of $u$ and $\bar{u}$ ) are relevant among all 
possible quartic interactions in $H_{4}$ because the corresponding fast oscillations exactly cancel out. The following expressions for the leading terms in (16) are obtained,

$$
\begin{gathered}
H_{2}(\eta, \xi)=\varepsilon \int \bar{u} \omega\left(k_{0}+\varepsilon D_{X}\right) u \mathrm{~d} X, \\
H_{4}(\eta, \xi)=\frac{\varepsilon^{3}}{4} \int\left[k_{0}^{3}|u|^{4}+\frac{3}{2} \varepsilon k_{0}^{2}|u|^{2}\left(\bar{u} D_{X} u+u \overline{D_{X} u}\right)\right] \mathrm{d} X, \\
H_{4}(-i \operatorname{sgn}(D) \eta, \xi)=-\frac{\varepsilon^{3}}{4} \int\left[k_{0}^{3}|u|^{4}+\frac{3}{2} \varepsilon k_{0}^{2}|u|^{2}\left(\bar{u} D_{X} u+u \overline{D_{X} u}\right)-2 \varepsilon k_{0}^{2}|u|^{2}\left|D_{X}\right||u|^{2}\right] \mathrm{d} X,
\end{gathered}
$$

up to fourth order in $\varepsilon$. We draw the reader's attention to the extra term in $H_{4}(-\mathrm{i} \operatorname{sgn}(D) \eta, \xi)$ as compared to $H_{4}(\eta, \xi)$, which will be further discussed below.

If we Taylor expand the dispersion relation $\omega\left(k_{0}+\varepsilon D_{X}\right)$ in $\varepsilon$, and retain terms of order up to $O\left(\varepsilon^{4}\right)$, then the Hamiltonian (16) further reduces to

$$
\begin{aligned}
H= & H_{2}(\eta, \xi)+H_{4}(\eta, \xi)-H_{4}(-i \operatorname{sgn}(D) \eta, \xi), \\
= & \frac{\varepsilon}{2} \int \bar{u}\left(\omega_{0}+\varepsilon \omega_{0}^{\prime} D_{X}+\frac{\varepsilon^{2}}{2} \omega_{0}^{\prime \prime} D_{X}^{2}+\frac{\varepsilon^{3}}{6} \omega_{0}^{\prime \prime \prime} D_{X}^{3}\right) u+\text { c.c. } \\
& \quad+\varepsilon^{2} k_{0}^{3}|u|^{4}+\frac{3}{2} \varepsilon^{3} k_{0}^{2}|u|^{2}\left(\bar{u} D_{X} u+u \overline{D_{X} u}\right)-\varepsilon^{3} k_{0}^{2}|u|^{2}\left|D_{X}\right||u|^{2} \mathrm{~d} X+O\left(\varepsilon^{5}\right), \\
= & \varepsilon \int \omega_{0}|u|^{2}+\varepsilon \omega_{0}^{\prime} \operatorname{Im}\left(\bar{u} \partial_{X} u\right)+\frac{\varepsilon^{2}}{2} \omega_{0}^{\prime \prime}\left|\partial_{X} u\right|^{2}+\frac{\varepsilon^{2}}{2} k_{0}^{3}|u|^{4} \\
& \quad+\frac{\varepsilon^{3}}{6} \omega_{0}^{\prime \prime \prime} \operatorname{Im}\left[\left(\overline{\partial_{X} u}\right)\left(\partial_{X}^{2} u\right)\right]+\frac{3}{2} \varepsilon^{3} k_{0}^{2}|u|^{2} \operatorname{Im}\left(\bar{u} \partial_{X} u\right) \\
& \quad-\frac{\varepsilon^{3}}{2} k_{0}^{2}|u|^{2}\left|D_{X}\right||u|^{2} \mathrm{~d} X+O\left(\varepsilon^{5}\right),
\end{aligned}
$$

where $\omega_{0}=\omega\left(k_{0}\right)=\sqrt{g k_{0}}$ and

$$
\omega_{0}^{\prime}=\frac{d \omega}{d k}\left(k_{0}\right)=\frac{\omega_{0}}{2 k_{0}}, \quad \omega_{0}^{\prime \prime}=\frac{d^{2} \omega}{d k^{2}}\left(k_{0}\right)=-\frac{\omega_{0}}{4 k_{0}^{2}}, \quad \omega_{0}^{\prime \prime \prime}=\frac{d^{3} \omega}{d k^{3}}\left(k_{0}\right)=\frac{3 \omega_{0}}{8 k_{0}^{3}} .
$$

Initials 'c.c.' in (21) stand for the complex conjugate of all the preceding terms on the right-hand side of this equation, and $\operatorname{Im}(\cdot)$ denotes the imaginary part.

We deduce from (20) that the evolution equation for $u$ is given by

$$
\begin{aligned}
\partial_{t} u= & -\mathrm{i} \varepsilon^{-1} \delta_{\bar{u}} H, \\
= & -\mathrm{i} \omega_{0} u-\varepsilon \omega_{0}^{\prime} \partial_{X} u+\mathrm{i} \frac{\varepsilon^{2}}{2} \omega_{0}^{\prime \prime} \partial_{X}^{2} u-\mathrm{i} \varepsilon^{2} k_{0}^{3}|u|^{2} u \\
& +\frac{\varepsilon^{3}}{6} \omega_{0}^{\prime \prime \prime} \partial_{X}^{3} u-3 \varepsilon^{3} k_{0}^{2}|u|^{2} \partial_{X} u+\mathrm{i} \varepsilon^{3} k_{0}^{2} u\left|D_{X}\right||u|^{2},
\end{aligned}
$$

which is a Hamiltonian counterpart to Dysthe's equation for deep-water gravity waves [1], associated with the Hamiltonian (21).

The prefactor $\varepsilon^{-1}$ in (20) should not be alarming because it suitably cancels out with the prefactor $\varepsilon$ in (21). The operator $D_{X}$ is substituted with $\partial_{X}=\mathrm{i} D_{X}$ wherever appropriate in (21) and (22). The cubic NLS equation is recovered if all $O\left(\varepsilon^{3}\right)$ terms in (22) are neglected. The nonlocal term, which involves the Fourier multiplier

$$
\left|D_{X}\right|=\operatorname{sgn}\left(D_{X}\right) D_{X}=-i \operatorname{sgn}\left(D_{X}\right) \partial_{X},
$$

in connection with the Hilbert transform, is a characteristic feature of Dysthe's equation and represents effects from the wave-induced mean flow [1,22]. By "mean flow", we refer to the lowest harmonic that is a slowly varying function of space. This nonlocal 
term naturally arises here from the expansion of $H_{4}(-\mathrm{i} \operatorname{sgn}(D) \eta, \xi)$ which is itself a direct consequence of the normal form transformation. It takes a simple explicit form, which differs from previous formulations of Dysthe's equation where it is coupled to the solution of an auxiliary Laplace problem for the unperturbed domain [4].

\subsection{Reconstruction of the Free Surface}

Because Equation (22) only solves for the wave envelope, another step is required in order to determine the actual shape of the free surface from knowledge of the wave envelope. In modulation theory, this reconstruction is typically a perturbative calculation in terms of a Stokes' expansion based on the modulational Ansatz. Contributions from up to the third harmonics of the wave spectrum are typically included in this expansion.

Our reconstruction procedure differs from the traditional method in that in inverts the transformations associated with our modulational Ansatz and with the third-order normal form. At any instant $t$, the original $\eta$ is recovered by solving the inviscid Burgers' equation

$$
\partial_{s} \widetilde{\eta}-\widetilde{\eta} \partial_{x} \widetilde{\eta}=0
$$

for $s \in(-1,0]$ with initial condition

$$
\left.\eta(x, t)\right|_{s=-1}=\frac{\varepsilon}{\sqrt{2}} a^{-1}(D)\left[u(X, t) e^{\mathrm{i} k_{0} x}+\bar{u}(X, t) e^{-\mathrm{i} k_{0} x}\right],
$$

where $u$ obeys (22) and $\eta$ is related to $\tilde{\eta}$ through (13). The choice of this initial condition is motivated by the special form of wave packets (17) and (19) we are modeling in this asymptotic regime. The final solution of (23) at $s=0$ is meant to represent the original physical variable $\eta$. From (24), higher-order contributions from lower (i.e., mean flow) and higher harmonics are generated through nonlinear interactions according to (23). The short interval $s \in(-1,0]$ ensures that the smooth and small initial condition (24) cannot yet develop into a shock.

As a consequence, the entire solution process including the surface reconstruction is carried out within the Hamiltonian framework. This approach was validated in [20] against direct numerical simulations of the full nonlinear Equations (6) and (7), as applied to the Benjamin-Feir instability of Stokes waves. The agreement was found to be slightly better than that obtained from predictions by the classical Dysthe's equation.

\section{Alternate Model for Spatial Dynamics}

\subsection{Envelope Equation}

In view of comparing with laboratory experiments where time series of the surface elevation are typically recorded by probes at fixed locations along the wave tank, much consideration has also been given to the spatial version of Dysthe's equation as indicated by the numerous studies in the literature [26-28]. Such a model is established by switching the role of the $X$ - and $t$-variables in the previous envelope equation, with $X$ acting as an evolutionary variable for wave propagation along the wave tank.

Because we are unaware of any Hamiltonian formulation for the spatial dynamics associated with the basic Equations (1)-(4), we proceed by applying the standard method to (22). Let

$$
v(X, t)=u(X, t) e^{i \omega_{0} t}
$$

so that

$$
\begin{aligned}
\partial_{X} v= & -2 \frac{\omega_{0}}{g \varepsilon} \partial_{t} v-\mathrm{i} \varepsilon \frac{g}{4 \omega_{0}^{2}} \partial_{X}^{2} v-2 \mathrm{i} \varepsilon \frac{\omega_{0} k_{0}^{3}}{g}|v|^{2} v \\
& +\varepsilon^{2} \frac{g^{2}}{8 \omega_{0}^{4}} \partial_{X}^{3} v-6 \varepsilon^{2} \frac{\omega_{0} k_{0}^{2}}{g}|v|^{2} \partial_{X} v+2 \mathrm{i} \varepsilon^{2} \frac{\omega_{0} k_{0}^{2}}{g} v\left|D_{X}\right||v|^{2}
\end{aligned}
$$


Then using (26) recursively, all $X$-derivatives on its right-hand side can be replaced by $t$-derivatives up to a certain order. This implies

$$
\begin{gathered}
\partial_{X}^{2} v=4 \frac{\omega_{0}^{2}}{g^{2} \varepsilon^{2}} \partial_{t}^{2} v+4 \mathrm{i} \frac{\omega_{0}}{g^{2} \varepsilon^{2}} \partial_{t}^{3} v+8 \mathrm{i} \frac{\omega_{0}^{2} k_{0}^{3}}{g^{2}} \partial_{t}\left(|v|^{2} v\right)+\ldots \\
\partial_{X}^{3} v=-8 \frac{\omega_{0}^{3}}{g^{3} \varepsilon^{3}} \partial_{t}^{3} v+\ldots
\end{gathered}
$$

and after inserting these expressions back in (26), we obtain

$$
\begin{aligned}
\partial_{X} v= & -2 \frac{\omega_{0}}{g \varepsilon} \partial_{t} v-\frac{\mathrm{i}}{g \varepsilon} \partial_{t}^{2} v-2 \mathrm{i} \varepsilon \frac{\omega_{0} k_{0}^{3}}{g}|v|^{2} v \\
& +16 \varepsilon \frac{k_{0}^{3}}{g}|v|^{2} \partial_{t} v+2 \varepsilon \frac{k_{0}^{3}}{g} v^{2} \partial_{t} \bar{v}+4 \mathrm{i} \varepsilon \frac{k_{0}^{3}}{g} v\left|D_{t}\right||v|^{2},
\end{aligned}
$$

which is the spatial version of (22). The coefficient $\varepsilon^{-1}$ in (27) can be removed by reintroducing the original spatial variable $x=X / \varepsilon$, which yields

$$
\begin{aligned}
\partial_{x} v= & -2 \frac{\omega_{0}}{g} \partial_{t} v-\frac{\mathrm{i}}{g} \partial_{t}^{2} v-2 \mathrm{i} \varepsilon^{2} \frac{\omega_{0} k_{0}^{3}}{g}|v|^{2} v \\
& +16 \varepsilon^{2} \frac{k_{0}^{3}}{g}|v|^{2} \partial_{t} v+2 \varepsilon^{2} \frac{k_{0}^{3}}{g} v^{2} \partial_{t} \bar{v}+4 \mathrm{i} \varepsilon^{2} \frac{k_{0}^{3}}{g} v\left|D_{t}\right||v|^{2} .
\end{aligned}
$$

The temporal counterpart to $D$ is defined by $D_{t}=i \partial_{t}$ so that its Fourier symbol is $\omega$. The variable $u$ is recovered by inverting (25). As is common for the spatial Dysthe's equation [26-28], there is no $\partial_{t}^{3} v$ term in (28). We note however an additional nonlinear term of the form $v^{2} \partial_{t} \bar{v}$ and, partly for this reason, the Hamiltonian nature of (22) is not retained by (28). This result is not viewed as undesirable because, as stated earlier, it does not contradict any basic Hamiltonian formulation for the spatial dynamics of water waves. Furthermore, because wave-tank experiments are usually susceptible to dissipative effects $[26,34,41]$, it is not clear whether a Hamiltonian model would be relevant in this context. Despite the absence of a conserved Hamiltonian, the $L^{2}$ norm (also called wave action)

$$
M=\int|v|^{2} \mathrm{~d} t
$$

is an invariant for the spatial dynamics of (28). This property can be proved by a direct calculation.

\subsection{Reconstruction of the Free Surface}

When using (28), it is also required to adjust the procedure for surface reconstruction accordingly. For (23), conversion of $\partial_{x} \widetilde{\eta}$ to $\partial_{t} \widetilde{\eta}$ is not straightforward given the complicated nature of (6) and (7). We use the approximation

$$
\partial_{x} \widetilde{\eta} \simeq-\frac{k_{0}}{\omega_{0}} \partial_{t} \widetilde{\eta}
$$

with $\tilde{\eta}$ obeying simple advection at the linear phase speed $\omega_{0} / k_{0}$. We choose (29) for right-moving solutions to be consistent with the advection part in (22). The new form of Burgers' equation is

$$
\partial_{s} \tilde{\eta}+\frac{k_{0}}{\omega_{0}} \tilde{\eta} \partial_{t} \widetilde{\eta}=0
$$

where

$$
\widetilde{\eta}=-\mathrm{i} \operatorname{sgn}(D) \eta \simeq-\mathrm{i} \operatorname{sgn}\left(D_{t}\right) \eta
$$


For the initial condition (24), we first Taylor expand it as

$$
\begin{aligned}
\left.\eta(x, t)\right|_{s=-1} & =\frac{\varepsilon}{\sqrt{2}}\left[e^{\mathrm{i} k_{0} x} a^{-1}\left(k_{0}+\varepsilon D_{X}\right) u(X, t)+e^{-\mathrm{i} k_{0} x} a^{-1}\left(-k_{0}+\varepsilon D_{X}\right) \bar{u}(X, t)\right], \\
& =\frac{\varepsilon}{\sqrt{2}}\left[e^{\mathrm{i} k_{0} x}\left(a_{0}^{-1}-\varepsilon a_{0}^{\prime} a_{0}^{-2} D_{X}\right) u+e^{-\mathrm{i} k_{0} x}\left(a_{0}^{-1}+\varepsilon a_{0}^{\prime} a_{0}^{-2} D_{X}\right) \bar{u}\right]+\ldots, \\
& =\frac{\varepsilon}{\sqrt{2}} a_{0}^{-1}\left(e^{\mathrm{i} k_{0} x} u+e^{-\mathrm{i} k_{0} x} \bar{u}\right)-\frac{\varepsilon^{2}}{\sqrt{2}} a_{0}^{\prime} a_{0}^{-2}\left(e^{\mathrm{i} k_{0} x} D_{X} u+e^{-\mathrm{i} k_{0} x} \overline{D_{X} u}\right), \\
& =\sqrt{2} \varepsilon a_{0}^{-1} \operatorname{Re}\left(e^{\mathrm{i} k_{0} x} u\right)-\sqrt{2} \varepsilon^{2} a_{0}^{\prime} a_{0}^{-2} \operatorname{Re}\left(e^{\mathrm{i} k_{0} x} D_{X} u\right),
\end{aligned}
$$

and then convert it to

$$
\left.\eta(x, t)\right|_{s=-1}=\sqrt{2} \varepsilon a_{0}^{-1} \operatorname{Re}\left(e^{\mathrm{i} \theta} v\right)-2 \sqrt{2} \varepsilon \frac{\omega_{0}}{g} a_{0}^{\prime} a_{0}^{-2} \operatorname{Re}\left[\mathrm{i} e^{\mathrm{i} \theta}\left(\partial_{t} v+\frac{\mathrm{i}}{2 \omega_{0}} \partial_{t}^{2} v\right)\right]+\ldots,
$$

by virtue of (25) and (27). For simplicity, we denote $a_{0}=a\left(k_{0}\right), a_{0}^{\prime}=a^{\prime}\left(k_{0}\right)$ and $\theta=$ $k_{0} x-\omega_{0} t$. Parity conditions imply that $a\left(k_{0}\right)=a\left(-k_{0}\right)$ and $a^{\prime}\left(-k_{0}\right)=-a^{\prime}\left(k_{0}\right)$, with

$$
a^{\prime}(k)=-\frac{a(k)}{4 k} .
$$

In this initial condition, the parameter $x$ would be assigned the location of a probe along the wave tank. The symbol $\operatorname{Re}(\cdot)$ denotes the real part.

The above expression of $\left.\eta(x, t)\right|_{s=-1}$ relies on the scale separation between $x$ and $X$, thus allowing the Fourier multiplier $a^{-1}(D)$ to be expanded up to first order in $\varepsilon$. Equation (27) is invoked to approximate the $X$-derivative of $v$ by $t$-derivatives. An alternate, perhaps simpler, approach consists in switching the $x$ - and $t$-variables directly in $a^{-1}(D)$ by virtue of (29), yielding

$$
\left.\eta(x, t)\right|_{s=-1}=\frac{\varepsilon}{\sqrt{2}} a^{-1}\left(\frac{k_{0}}{\omega_{0}} D_{t}\right)\left(v e^{\mathrm{i} \theta}+\bar{v} e^{-\mathrm{i} \theta}\right),
$$

where $v$ solves (28). In general, the latter option should be preferred because it does not involve any expansion of $a^{-1}(D)$.

\subsection{Rescaled Variables}

We now consider unscaled versions of (28)-(31) where the perturbation parameter $\varepsilon$ is absorbed back into the variables. To facilitate the comparison with wave-channel measurements, we change to a coordinate system moving at the linear group velocity via the dimensionless rescaled variables

$$
\eta=A_{0} \zeta, \quad v=B_{0} V, \quad \chi=\varepsilon^{2} k_{0} x, \quad \tau=\varepsilon \gamma \omega_{0}\left(\frac{2 \omega_{0}}{g} x-t\right),
$$

where $\gamma$ is a scale factor that fits the computational domain into an interval of length $2 \pi$ and $B_{0}$ is a characteristic (real-valued) amplitude for the wave envelope such that

$$
B_{0}=A_{0} \sqrt[4]{\frac{g}{4 k_{0}}}=\frac{1}{\sqrt{2}} a_{0} A_{0},
$$

according to (17) and (19). This change of variables reintroduces $\varepsilon$ in the same way as proposed by Lo and Mei [26], and will also allow us to compare our results with their model, which may be viewed as a spatial form of the classical (non-Hamiltonian) Dysthe's equation. Recall that the wave steepness is defined by $\varepsilon=k_{0} A_{0}$, hence $k_{0}^{2} B_{0}^{2}=\varepsilon^{2} a_{0}^{2} / 2$, and the group velocity is given by $\omega_{0}^{\prime}=g /\left(2 \omega_{0}\right)$ for gravity waves on deep water. To a fixed observer, $\tau$ denotes the negative time elapsed and $\chi$ represents the distance over which the 
wave group has advanced (i.e., the fetch). In terms of these new variables, the envelope equation (28) becomes

$$
\begin{aligned}
\partial_{\chi} V= & -\mathrm{i} \gamma^{2} \partial_{\tau}^{2} V-\mathrm{i} \beta|V|^{2} V-8 \varepsilon \gamma \beta|V|^{2} \partial_{\tau} V \\
& -\varepsilon \gamma \beta V^{2} \partial_{\tau} \bar{V}+2 \mathrm{i} \varepsilon \gamma \beta V\left|D_{\tau}\right||V|^{2}
\end{aligned}
$$

where $\beta=\omega_{0} a_{0}^{2} / g=1$. The wave envelope is now represented by a complex function $V(\tau, \chi)$ of $\tau$ and $\chi$. For the surface reconstruction, Burgers' equation (30) takes the dimensionless form

$$
\partial_{s} \widetilde{\zeta}-\varepsilon^{2} \gamma \widetilde{\zeta} \partial_{\tau} \widetilde{\zeta}=0
$$

with initial condition

$$
\left.\zeta(\tau, \chi)\right|_{s=-1}=\operatorname{Re}\left(e^{\mathrm{i} \theta} V\right)+2 \varepsilon \gamma k_{0} a_{0}^{\prime} a_{0}^{-1} \operatorname{Re}\left(\mathrm{i} e^{\mathrm{i} \theta} \partial_{\tau} V\right),
$$

up to order $O(\varepsilon)$, where the phase function now reads $\theta=\tau /(\varepsilon \gamma)-\chi / \varepsilon^{2}$. The alternate choice (31) reduces to

$$
\left.\zeta(\tau, \chi)\right|_{s=-1}=\frac{1}{2}(\varepsilon \gamma)^{1 / 4}\left|D_{\tau}\right|^{1 / 4}\left(e^{\mathrm{i} \theta} V+e^{-\mathrm{i} \theta} \bar{V}\right),
$$

after substituting

$$
a^{-1}\left(\frac{k_{0}}{\omega_{0}} D_{t}\right)=a^{-1}\left(-\varepsilon \gamma k_{0} D_{\tau}\right),
$$

with its definition (18). Note that the Fourier multiplier $\left|D_{\tau}\right|^{1 / 4}$ acts on all the functions inside the parentheses in (35). For the tests considered in the next section, we found that (34) and (35) produce similar results and we opted to present those given by (35).

\section{Numerical Results}

Validation of the Hamiltonian Dysthe's equation (22) was provided in [20] by comparing with time-domain simulations of the full Equations (6) and (7) and of the classical Dysthe's equation. In the present study, we focus on the spatial version (28) and test it against laboratory experiments by Keller [42] on periodic groups and by Su [43] on short-wave packets. These two different sets of experiments were discussed by Lo and Mei [26] and were used to validate their spatial version of the classical Dysthe's equation. We follow their procedure as described in the previous section, and take this opportunity to compare with their model as well. The latter takes the dimensionless form

$$
\partial_{\chi} A=-\mathrm{i} \gamma^{2} \partial_{\tau}^{2} A-\mathrm{i}|A|^{2} A-8 \varepsilon \gamma|A|^{2} \partial_{\tau} A+2 \mathrm{i} \varepsilon \gamma A\left|D_{\tau}\right||A|^{2},
$$

in terms of the same rescaled variables $\tau$ and $\chi$ as defined earlier. In this case (as is common in modulation theory), the free surface is reconstructed perturbatively via the Stokes' expansion

$$
\zeta(\tau, \chi)=\frac{1}{2}\left[A_{1}(\tau, \chi) e^{\mathrm{i} \theta}+A_{2}(\tau, \chi) e^{2 \mathrm{i} \theta}+A_{3}(\tau, \chi) e^{3 \mathrm{i} \theta}\right]+\text { c.c. }+A_{0}(\tau, \chi),
$$

up to the third harmonics, where

$$
\begin{gathered}
A_{0}(\tau, \chi)=-\frac{1}{2} \varepsilon^{2} \gamma\left|D_{\tau}\right||A|^{2}, \quad A_{1}(\tau, \chi)=A-\mathrm{i} \varepsilon \gamma \partial_{\tau} A-\frac{3}{8} \varepsilon^{2}|A|^{2} A, \\
A_{2}(\tau, \chi)=\frac{1}{2} \varepsilon A^{2}-2 \mathrm{i} \varepsilon^{2} \gamma A \partial_{\tau} A, \quad A_{3}(\tau, \chi)=\frac{3}{8} \varepsilon^{2} A^{3} .
\end{gathered}
$$




\subsection{Numerical Methods}

Equation (32) is discretized in $\tau$ by a pseudo-spectral method based on the fast Fourier transform $\mathcal{F}$. The computational domain spans the interval $0 \leq \tau \leq 2 \pi$ with periodic boundary conditions and is divided into a regular grid of $N$ collocation points. We denote the discrete Fourier coefficients $\widehat{U}(\kappa)$ of a $2 \pi$-periodic function $U(\tau)$ by

$$
\widehat{U}(\kappa)=\mathcal{F}\{U(\tau)\}(\kappa), \quad \kappa=0, \pm 1, \pm 2, \ldots, \pm N / 2,
$$

where $\kappa$ represents the sideband wavenumbers associated with the variable $\tau$. Integration over $\chi$ is carried out in the Fourier space so that the linear terms are solved exactly by the integrating factor technique. The nonlinear terms are integrated in $\chi$ by using a fourth-order Runge-Kutta scheme with constant step $\Delta \chi$.

The same numerical methods are used to solve Burgers' Equation (33) with initial condition (35) for the surface reconstruction. The reader is referred to $[9,20,23,36]$ for more details on such simulations. In particular, Equation (33) is integrated over $s$ by using the same step size $\Delta s=\Delta \chi$. We remark that, although the surface reconstruction requires solving an additional nonlinear PDE, this calculation is relatively straightforward and is not necessarily performed at each downstream location $\chi$ (only when data on the free surface are needed at the post-processing stage, e.g., for plotting purposes). Furthermore, because this PDE is solved over a relatively short interval $s \in(-1,0]$, the associated cost is insignificant and does not undermine the overall efficiency of this modulational approach. The whole computation (i.e. solving for the wave envelope and determining the surface elevation) can be produced by a single self-contained computer program.

Equations (36) and (37) from Lo and Mei [26] are computed by the same numerical strategy, with exactly the same values for the numerical parameters.

\subsection{Comparison with Experiments on Periodic Groups}

For small amplitudes, the superposition of two progressive waves with slightly different frequencies gives rise to periodic groups whose envelopes move forward at the group velocity without change of shape. Keller [42] conducted laboratory experiments for such groups with maximum incident steepness $\varepsilon$ up to 0.23 . He found that each group tended to lean forward.

To mimic Keller's input data, Lo and Mei [26] prescribed the incident condition

$$
A(\tau, 0)=0.483 e^{\mathrm{i} \tau}+0.537 e^{-\mathrm{i} \tau},
$$

at $\chi=0$ for (36), so that $\left|\widehat{A}_{1}( \pm 1,0)\right|=0.5$ for the two parent sidebands $(\kappa= \pm 1)$ of the first harmonic $A_{1}(\tau, 0)$, as in the laboratory experiment. The initial values of the higher sidebands were small and are neglected in (38). The physical parameters are $g=9.81 \mathrm{~m}$ $\mathrm{s}^{-2}, k_{0}=8.865 \mathrm{~m}^{-1}$ and $\varepsilon=k_{0} A_{0}=0.23$. The carrier frequency is estimated by the linear dispersion relation $\omega_{0}=\sqrt{g k_{0}}$. Similarly, we specify the incident condition

$$
V(\tau, 0)=0.494 e^{\mathrm{i} \tau}+0.507 e^{-\mathrm{i} \tau},
$$

for (32), so that

$$
\left|\widehat{V}_{1}( \pm 1,0)\right|=(\varepsilon \gamma)^{1 / 4}\left|\mathcal{F}\left\{e^{-\mathrm{i} \theta}\left|D_{\tau}\right|^{1 / 4}\left(e^{\mathrm{i} \theta} V\right)\right\}( \pm 1,0)\right|=0.5,
$$

based on the expression (35) of $\left.\zeta(\tau, \chi)\right|_{s=-1}$. Here we deem the first harmonic $V_{1}(\tau, \chi)$ (and its sidebands) to be well approximated in this way because, as suggested by the scaling factor $\varepsilon^{2}$ of the nonlinear term, Equation (33) is expected to only produce small contributions to wave harmonics of the free surface over the short interval $s \in(-1,0]$. We also point out that, because the variables $A$ and $V$ do not quite represent the same physical quantity, the numerical coefficients in (38) and (39) are not expected to be identical. 
As shown in Figure 1, the computed surface displacements $\zeta$ compare favorably with the measurements (which are extracted from Figure 1 in [26]). These are limited sets of data for crests and troughs that were recorded at eight stations within $7.14 \mathrm{~m}$ from the wavemaker. Overall, the phase and asymmetry of the wave groups, which tend to lean forward, are well reproduced. Discrepancies are noticeable and may be attributed to experimental errors, physical effects that are not taken into account in the theory (e.g., friction, local wave breaking, undertow current in the wave tank), or uncertainty in the input data. Predictions of $\zeta$ based on our simulations of (32) and (36) are also compared in Figure 1. Recall that the free surface is reconstructed via (33) with (35) for our model (32), while it is determined by (37) for the spatial form (36) of the classical Dysthe's equation. The numerical parameters are $\gamma=0.229, N=256$ and $\Delta \chi=0.01$. As expected, the two numerical solutions are indistinguishable (at least to graphical scale) near the wavemaker. They remain close together, although small discrepancies tend to develop around the dominant crest as the wave group travels down the tank. Model (32) seems to agree slightly better with the experiment, as indicated e.g., at $x=6 \mathrm{~m}$ (crest at $\tau \simeq 3.8$ ) and at $x=6.57 \mathrm{~m}$ (trough at $\tau \simeq 3.9$ ).
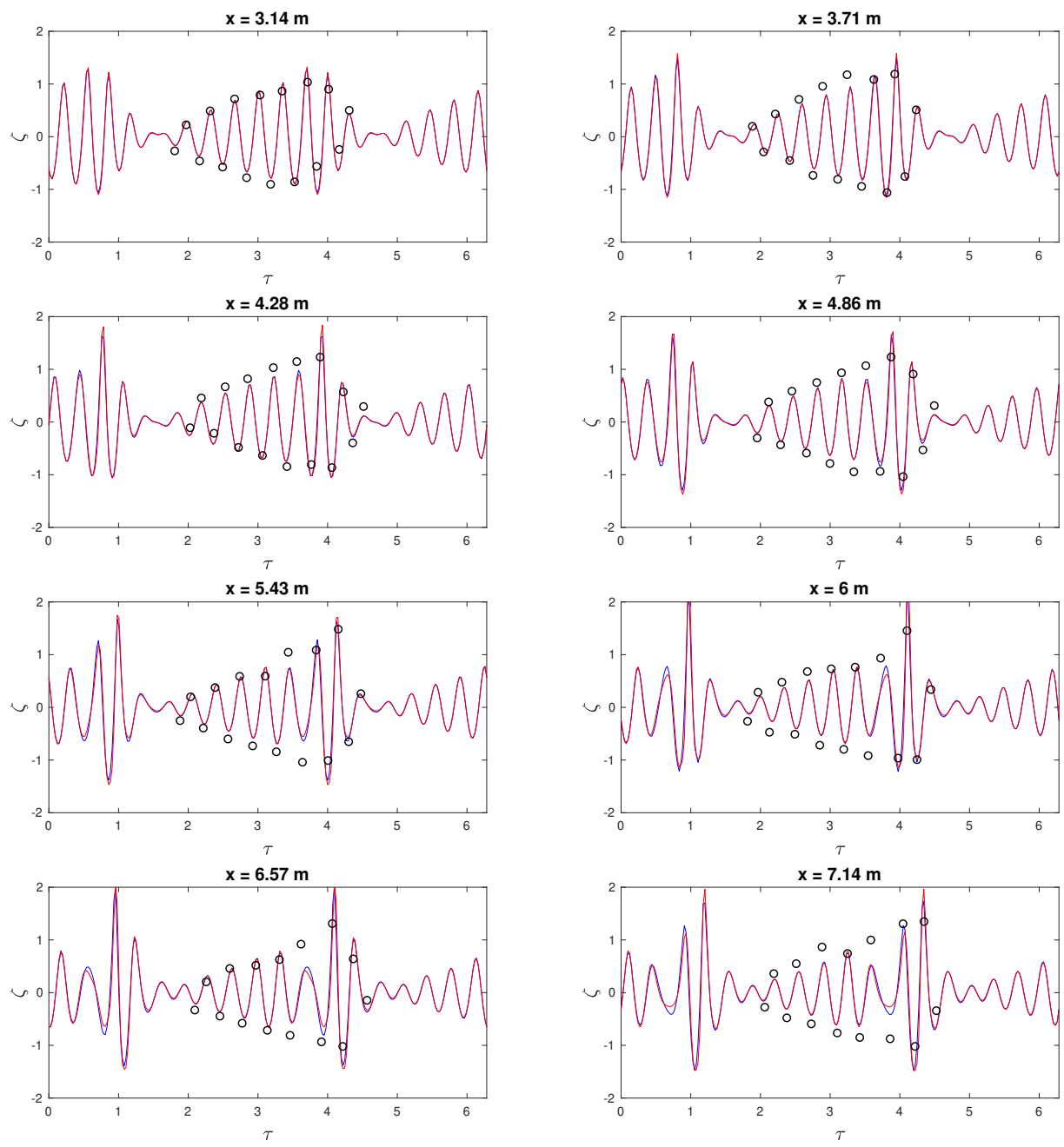

Figure 1. Measured (circles) and computed (lines) surface displacements at 8 stations along the wave tank in Keller's experiments [42] on periodic groups. The parameters are $\gamma=0.229, \varepsilon=0.23$, $k_{0}=8.865 \mathrm{~m}^{-1}$. Predictions from our model (blue line) and the spatial form of the classical Dysthe's equation (red line) are compared. The positions $x$ of the probes are $3.14 \mathrm{~m}, 3.71 \mathrm{~m}, 4.28 \mathrm{~m}, 4.86 \mathrm{~m}$, $5.43 \mathrm{~m}, 6.00 \mathrm{~m}, 6.57 \mathrm{~m}, 7.14 \mathrm{~m}$. 
Figure 2a shows the evolution of the parent sidebands $(\kappa= \pm 1)$ and higher sidebands $\kappa= \pm 3$ for the first harmonic of the free surface, again comparing predictions from (32) with Keller's measurements (which are extracted from Figure 2 in [26]). The agreement is good overall and is similar to that found for (36) as illustrated in Figure 2b. By contrast, predictions from the cubic NLS equation (by neglecting the higher-order nonlinear terms in Equation (32)) are plotted in Figure 2c and are clearly unsatisfactory. In particular, the corresponding sidebands $\kappa=+1$ and +3 behave quite differently from the measurements.

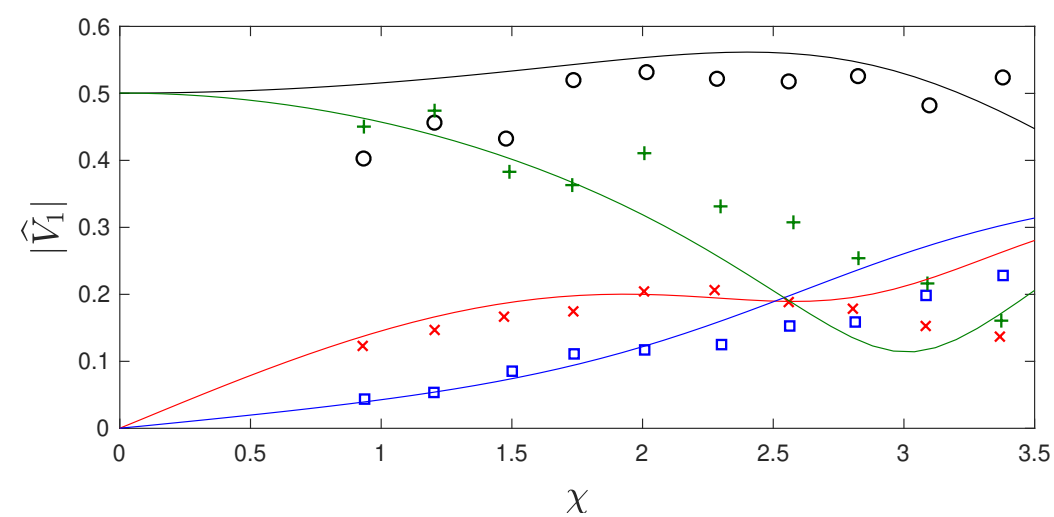

(a)

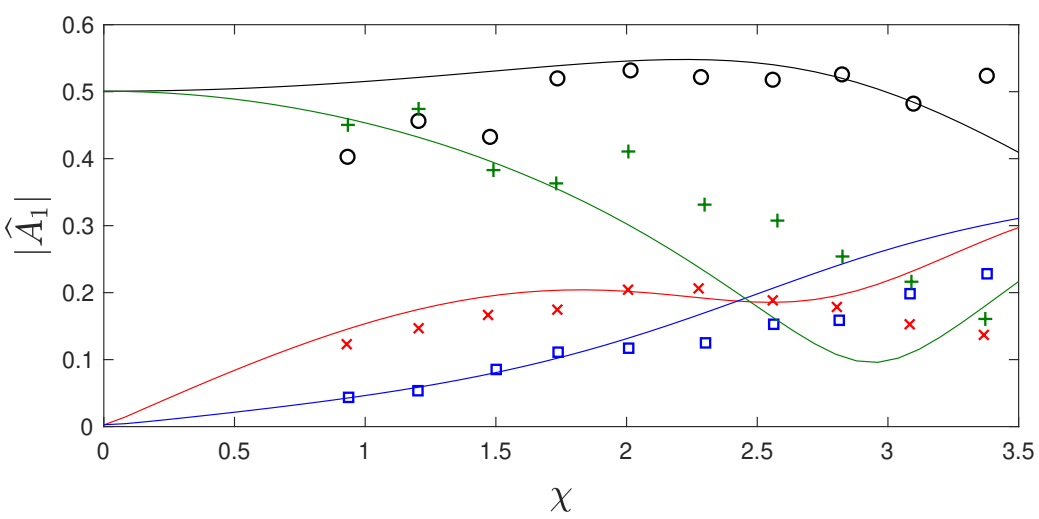

(b)

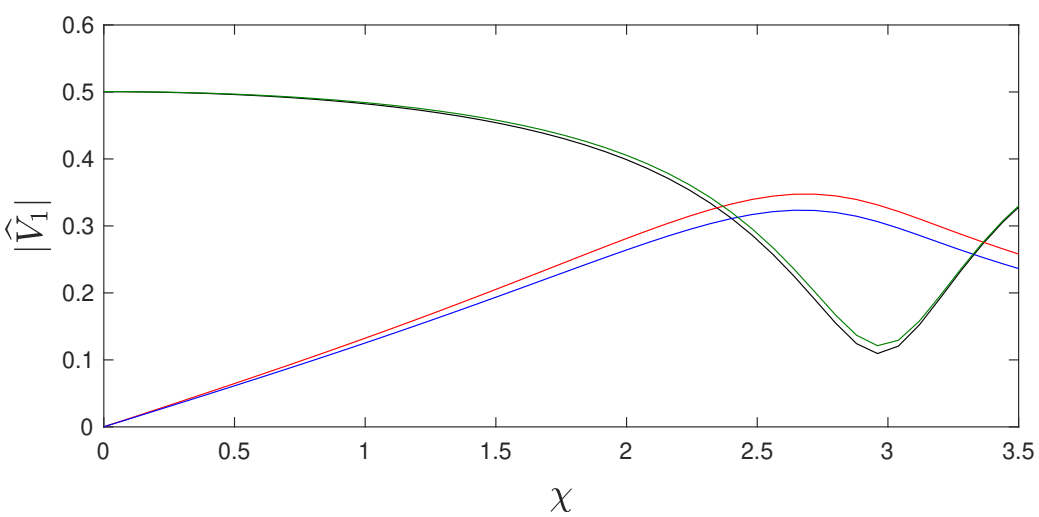

(c)

Figure 2. Measured (symbols) and computed (lines) sideband amplitudes of the first harmonic for Keller's experiment [42] as shown in Figure 1: $\kappa=-1$ (green), $\kappa=+1$ (black), $\kappa=-3$ (red), $\kappa=+3$ (blue). The panels correspond to (a) our model (32), (b) spatial form (36) of the classical Dysthe's equation, (c) cubic nonlinear Schrödinger (NLS) model. 
Figure 3 depicts the evolution of the same sidebands for a duration much longer than that of the experiment. Again, the similarity in results between (32) and (36) persists. We can recognize a near-recurrence phenomenon as often reported in such situations [44]. All these results on surface profiles and spectral amplitudes for periodic wave groups are consistent with Lo and Mei's observations (see Figures 1-3 in [26]). Finally, the evolution of the relative error

$$
\frac{\Delta M}{M_{0}}=\frac{\left|M-M_{0}\right|}{M_{0}},
$$

on the rescaled wave action

$$
M=\int|U|^{2} \mathrm{~d} \tau,
$$

for (32) $(U=V)$ and for (36) $(U=A)$ is illustrated in Figure 4. The integral is computed via the trapezoidal rule over the periodic interval $[0,2 \pi]$. The reference value $M_{0}$ denotes the incident value of (40) at $\chi=0$. Overall, $M$ is well conserved in both cases, despite a gradual loss of accuracy that is partly due to accumulation of numerical errors. Model (32) seems to perform slightly better in this regard, displaying a slightly lower error overall. Note that, although filtering was not used for the tests in Figures 1 and 2, it was needed for the tests in Figures 3 and 4 (using an exponential low-pass filter as in [9]) so that the numerical solution can be computed over the longer interval $\chi \in[0,15]$ given the relatively large incident steepness $\varepsilon=0.23$. Use of filtering likely contributes to the loss of accuracy as seen in Figure 4. We emphasize that the same filter together with the same values of numerical parameters were specified in the simulations of (32) and (36), and we point out that Lo and Mei [26] also used filtering.

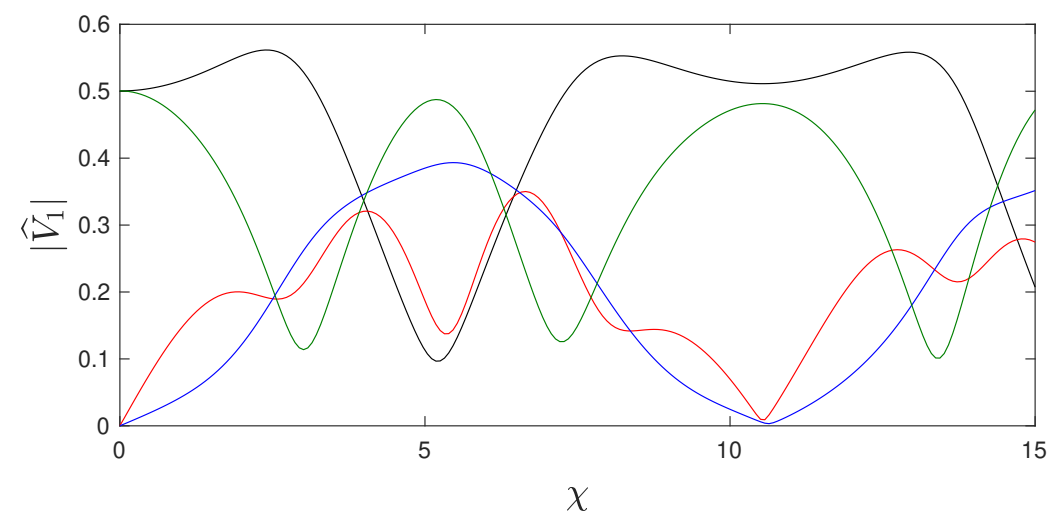

(a)

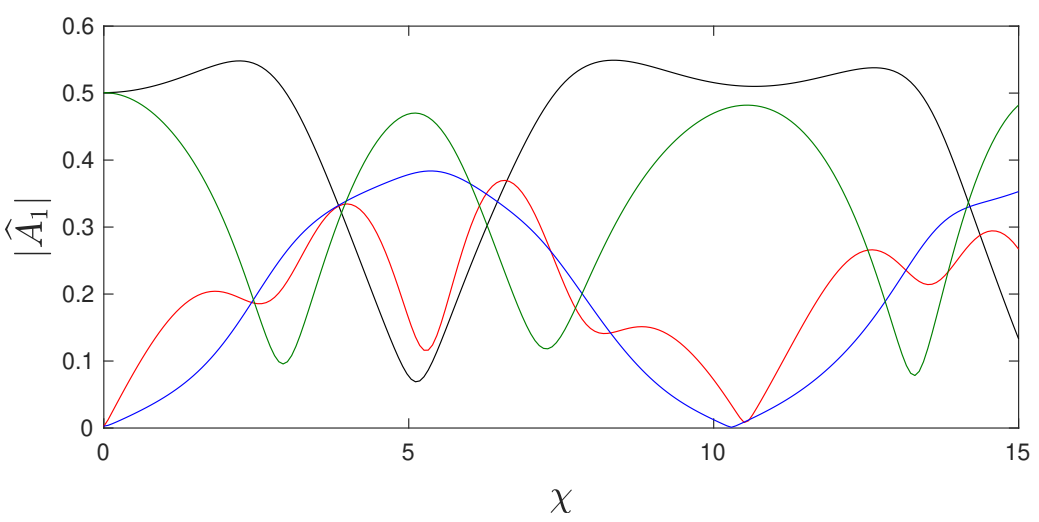

(b)

Figure 3. Computed sideband amplitudes of the first harmonic over a longer $\chi$-interval for the same parameter values and same color notations as in Figure 2. The panels correspond to (a) our model (32), (b) spatial form (36) of the classical Dysthe's equation. 


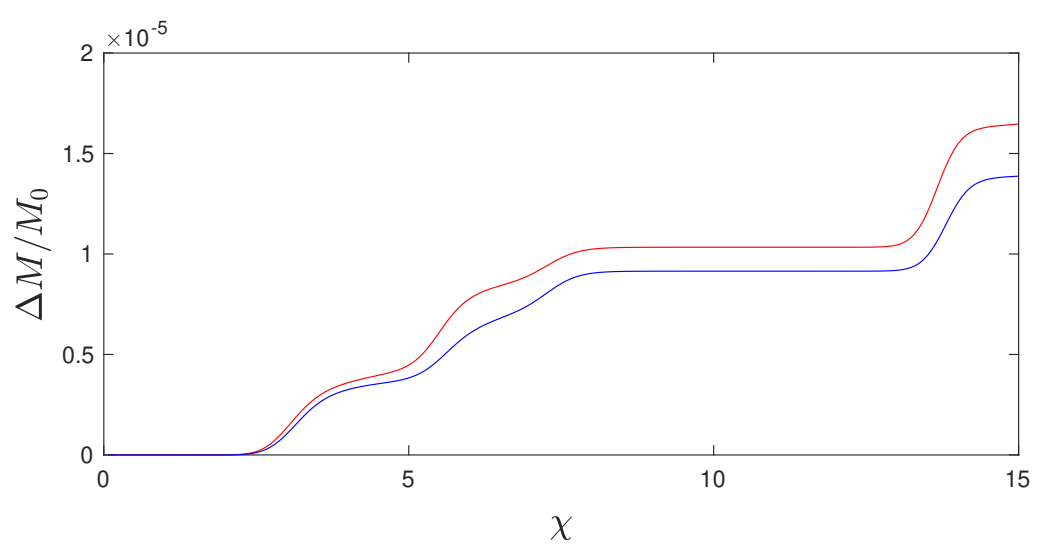

Figure 4. Relative error on $M$ for the same parameter values as in Figure 2. Predictions from our model (blue line) and the spatial form of the classical Dysthe's equation (red line) are compared.

\subsection{Comparison with Experiments on Short-Wave Packets}

Feir [45] performed laboratory experiments for packets of bell-shaped envelope and of roughly equal duration but different maximum amplitudes. For low wave slope, the envelopes remained symmetrical and flattened with distance. For relatively steep waves however, the envelopes first steepened forward and then split into two groups with the smaller group trailing behind. More extensive experiments in a long tank for initially square envelopes with maximum incident steepness $\varepsilon=0.09-0.28$ were conducted by $\mathrm{Su}$ [43]. Surface displacements were recorded at eight stations within $106.7 \mathrm{~m}$ from the wavemaker. Su [43] confirmed Feir's results on the asymmetry of evolution and further revealed that the separated groups have different frequencies.

We consider a case that was investigated by Su [43] and, following Lo and Mei [26], the incident condition for (36) is taken to be

$$
A\left(\tau_{j}, 0\right)=C\left[\tanh \left(j-j_{a}\right)-\tanh \left(j-j_{b}\right)\right], \quad j=1,2, \ldots, N,
$$

where $C=0.5$ and $j_{a}=124 \mathrm{~N} / 256, j_{b}=134 \mathrm{~N} / 256$. As so defined, the edges are slightly smoothed to avoid numerical instability. We specify the incident condition $V(\tau, 0)$ for (32) to be of the same form as (41) with the exception that $C=0.495$. In this way, the maximum amplitude of $\left.\zeta(\tau, 0)\right|_{s=0}$ via (33) coincides with the maximum amplitude of $\zeta(\tau, 0)$ from (37).

Figure 5 shows the computed free surface $\zeta$ at four stations along the wave tank for $\varepsilon=0.09$ and $\omega_{0} /(2 \pi)=0.96 \mathrm{~Hz}$. The carrier wavenumber is estimated by inverting the linear dispersion relation $k_{0}=\omega_{0}^{2} / g$. Wave profiles from our simulations of (32) and (36) are again compared in this figure. Discrepancies are barely noticeable for as far as $x=106.7$ $\mathrm{m}$. The numerical parameters are $\gamma=0.0868, N=1024, \Delta \chi=0.001$ and filtering was not needed here. These results closely resemble the computations presented in Figure 5 from [26]. Note the slightly asymmetric envelope between $x=42.7 \mathrm{~m}$ and $x=91.5 \mathrm{~m}$, which is also visible in Su's records. The wave period is longer in the lower group in front of the main group. The backward leaning of the main group is associated with nonlinear modification of the dispersion relation, as pointed out in [26]. A more quantitative analysis is provided in Table 1 where predictions of the maximum local steepness $k_{0} A_{m}$ based on our simulations of (32) and (36) are compared to Su's measurements at all eight stations (which are reproduced from Table 1 in [26]). The agreement is good overall. The computations tend to overestimate the maximum local steepness as the wave travels down the tank, which is expected considering that dissipation is not accounted for in the theory. Indeed, dissipative effects on wave propagation are likely to be relevant for such a long tank as used in [43]. Compared to (36), predictions from (32) are found to be slightly closer to the experimental data at the three stations farthest downstream. 

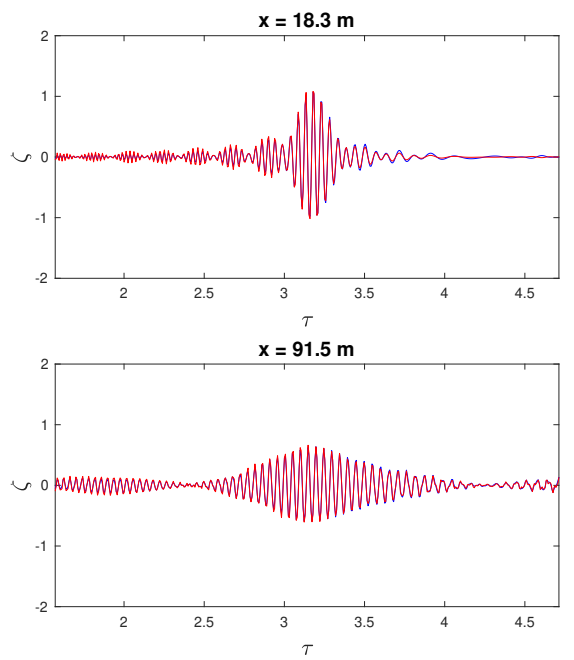
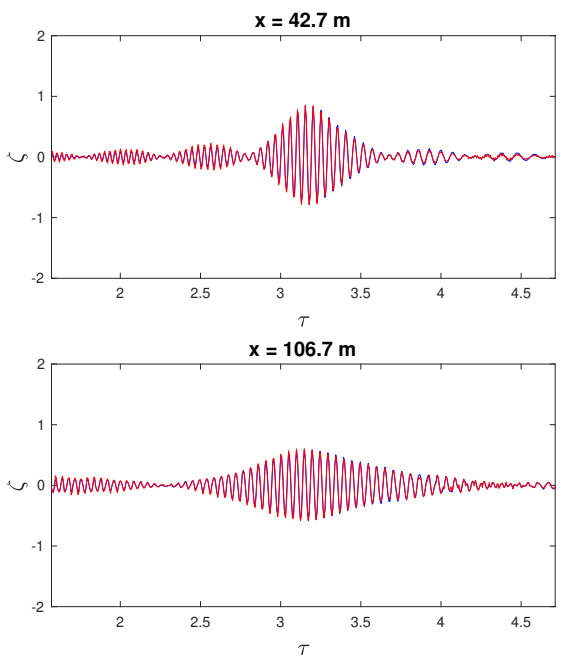

Figure 5. Computed surface displacements at four stations along the wave tank in Su's experiments [42] on short-wave packets. The parameters are $\gamma=0.0868, \varepsilon=0.09, \omega_{0} /(2 \pi)=0.96 \mathrm{~Hz}$. Predictions from our model (blue line) and the spatial form of the classical Dysthe's equation (red line) are compared. The positions $x$ of the probes are $18.3 \mathrm{~m}, 42.7 \mathrm{~m}, 91.5 \mathrm{~m}, 106.7 \mathrm{~m}$.

Table 1. Measured and computed maximum local steepness $k_{0} A_{m}$ at eight stations along the wave tank in Su's experiments [43] on short-wave packets. The parameters are $\gamma=0.0868, \varepsilon=0.09$, $\omega_{0} /(2 \pi)=0.96 \mathrm{~Hz}$. Predictions from our model (third column) and the spatial form of the classical Dysthe's equation (fourth column) are reported.

\begin{tabular}{cccc}
\hline $\boldsymbol{x}(\mathbf{m})$ & Measured & $\begin{array}{c}\text { Computed } \\
\text { (Equation (32)) }\end{array}$ & $\begin{array}{c}\text { Computed } \\
\text { (Equation (36)) }\end{array}$ \\
\hline 6.1 & 0.107 & 0.123 & 0.125 \\
18.3 & 0.120 & 0.096 & 0.097 \\
24.4 & 0.094 & 0.086 & 0.089 \\
42.7 & 0.073 & 0.075 & 0.077 \\
61.0 & 0.066 & 0.065 & 0.066 \\
76.3 & 0.053 & 0.059 & 0.060 \\
91.5 & 0.049 & 0.057 & 0.059 \\
106.7 & 0.047 & 0.052 & 0.054 \\
\hline
\end{tabular}

\section{Conclusions}

We have given an overview of the Hamiltonian modulational approach that has been advocated in a series of papers by Craig et al. [21-25] and which leads to a Hamiltonian version of Dysthe's equation for two-dimensional gravity waves on deep water, as recently shown in [20]. We have described the main steps in the derivation of the evolution equation for the complex wave envelope, together with the procedure to reconstruct the free surface from this wave envelope. Both operations are performed within the Hamiltonian framework, which ensures some consistency throughout the entire solution process. A key ingredient in this approach is a Birkhoff normal form transformation that eliminates all non-resonant cubic terms, and yields a simple and elegant expression for the reduced Hamiltonian. As a consequence of this reduction, the surface reconstruction is a non-perturbative calculation but requires solving a nonlinear PDE which, in this problem, takes the generic form of an inviscid Burgers' equation.

To facilitate comparison with laboratory experiments, an alternate spatial form of this Hamiltonian model can be written. This involves switching the role of the spatial and temporal variables in the equations governing the envelope evolution and the surface reconstruction. We have proposed an improved strategy for the latter computation, where a refined choice of the initial condition is specified to solve Burgers' equation. Focusing on 
the spatial dynamics and following Lo and Mei [26], we have introduced a dimensionless rescaled form of these equations and performed numerical tests against laboratory experiments by Keller [42] for periodic groups and by Su [43] for short-wave packets. Satisfactory agreement is obtained in all these tests. We have also validated our numerical results by comparing with the spatial form of the classical Dysthe's equation. Predictions from our new model and its classical counterpart are found to be similar in all the cases considered.

It would be of interest to extend the present methods and models to the finite-depth case as well as to the three-dimensional setting. Preliminary results in these directions based on the DNO can be found in [46]. These problems pose additional challenges and their study is envisioned for future work.

Author Contributions: Investigation, P.G., A.K. and C.S.; software, P.G. and B.X.; supervision, P.G. and C.S.; writing-review and editing, P.G., A.K., C.S. and B.X. All authors have read and agreed to the published version of the manuscript.

Funding: Adilbek Kairzhan thanks the Fields Institute for Research in Mathematical Sciences for its support and hospitality during the thematic program on Mathematical Hydrodynamics. Catherine Sulem is partially supported by the Natural Sciences and Engineering Research Council of Canada (grant number 2018-04536) and a Killam Research Fellowship from the Canada Council for the Arts.

Acknowledgments: This paper is dedicated to the memory of our dear friend Walter Craig, with admiration and gratitude.

Conflicts of Interest: The authors declare no conflict of interest.

\section{Abbreviations}

The following abbreviations are used in this manuscript:

DNO Dirichlet-Neumann operator

NLS Nonlinear Schrödinger equation

PDE Partial differential equation

\section{References}

1. Dysthe, K.B. Note on a modification to the nonlinear Schrödinger equation for application to deep water waves. Proc. R. Soc. Lond. A 1979, 369, 105-114.

2. Brinch-Nielsen, U.; Jonsson, I.G. Fourth order evolution equations and stability analysis for Stokes waves on arbitrary water depth. Wave Motion 1986, 8, 455-472. [CrossRef]

3. Hogan, S.J. The fourth-order evolution equation for deep-water gravity-capillary waves. Proc. R. Soc. Lond. A 1985, 402, 359-372. [CrossRef]

4. Trulsen, K.; Kliakhandler, I.; Dysthe, K.B.; Velarde, M.G. On weakly nonlinear modulation of waves on deep water. Phys. Fluids 2000, 12, 2432-2437. [CrossRef]

5. Hara, T.; Mei, C.C. Frequency downshift in narrowbanded surface waves under the influence of wind. J. Fluid Mech. 1991, 230, 429-477. [CrossRef]

6. Slunyaev, A.; Pelinovsky, E. Numerical simulations of modulated waves in a higher-order Dysthe equation. Water Waves 2020, 2, 59-77. [CrossRef]

7. Craig, W.; Sulem, C. Numerical simulation of gravity waves. J. Comput. Phys. 1993, 108, 73-83. [CrossRef]

8. Guyenne, P.; Grilli, S.T. Numerical study of three-dimensional overturning waves in shallow water. J. Fluid Mech. 2006, 547, 361-388. [CrossRef]

9. Xu, L.; Guyenne, P. Numerical simulation of three-dimensional nonlinear water waves. J. Comput. Phys. 2009, $228,8446-8466$. [CrossRef]

10. Zakharov, V.E.; Dyachenko, A.I.; Vasilyev, O.A. New method for numerical simulation of a nonstationary potential flow of incompressible fluid with a free surface. Eur. J. Mech. B Fluids 2002, 21, 283-291. [CrossRef]

11. Zakharov, V.E. Stability of periodic waves of finite amplitude on the surface of a deep fluid. J. Appl. Mech. Tech. Phys. 1968, 9, 190-194. [CrossRef]

12. Krasitskii, V.P. On reduced equations in the Hamiltonian theory of weakly nonlinear surface waves. J. Fluid Mech. 1994, 272, 1-20. [CrossRef]

13. Stiassnie, M. Note on the modified nonlinear Schrödinger equation for deep water waves. Wave Motion 1984, 6, 431-433. [CrossRef]

14. Grande, R.; Kurianski, K.M.; Staffilani, G. On the nonlinear Dysthe equation. arXiv 2020, arXiv:2006.13392. 
15. Mosincat, R.; Pilod, D.; Saut, J.-C. Global well-posedness and scattering for the Dysthe equation. J. Math. Pures Appl. 2021. [CrossRef]

16. Düll, W.-P.; Schneider, G.; Wayne, C.E. Justification of the nonlinear Schrödinger equation for the evolution of gravity driven 2D surface water waves in a canal of finite depth. Arch. Ration. Mech. Anal. 2016, 220, 543-602. [CrossRef]

17. Totz, N.; Wu, S. A rigorous justification of the modulation approximation to the $2 \mathrm{D}$ full water wave problem. Commun. Math Phys. 2012, 310, 817-883. [CrossRef]

18. Sulem, C.; Sulem, P.-L. The Nonlinear Schrödinger Equation: Self-Focusing and Wave Collapse; Springer: New York, NY, USA, 1999.

19. Gramstad, O.; Trulsen, K. Hamiltonian form of the modified nonlinear Schrödinger equation for gravity waves on arbitrary depth. J. Fluid Mech. 2011, 670, 404-426. [CrossRef]

20. Craig, W.; Guyenne, P.; Sulem, C. Normal form transformations and Dysthe's equation for the nonlinear modulation of deep-water gravity waves. Water Waves 2021, 3. [CrossRef]

21. Craig, W.; Guyenne, P.; Nicholls, D.P.; Sulem, C. Hamiltonian long-wave expansions for water waves over a rough bottom. Proc. R. Soc. Lond. A 2005, 461, 839-873. [CrossRef]

22. Craig, W.; Guyenne, P.; Sulem, C. A Hamiltonian approach to nonlinear modulation of surface water waves. Wave Motion 2010, 47, 552-563. [CrossRef]

23. Craig, W.; Guyenne, P.; Sulem, C. Hamiltonian higher-order nonlinear Schrödinger equations for broader-banded waves on deep water. Eur. J. Mech. B Fluids 2012, 32, 22-31. [CrossRef]

24. Craig, W.; Guyenne, P.; Sulem, C. The surface signature of internal waves. J. Fluid Mech. 2012, 710, 277-303. [CrossRef]

25. Craig, W.; Sulem, C. Mapping properties of normal forms transformations for water waves. Boll. Unione Mat. Ital. 2016, 9, $289-318$. [CrossRef]

26. Lo, E.; Mei, C.C. A numerical study of water-wave modulation based on a higher-order nonlinear Schrödinger equation. J. Fluid Mech. 1985, 150, 395-416. [CrossRef]

27. Shemer, L.; Kit, E.; Jiao, H. An experimental and numerical study of the spatial evolution of unidirectional nonlinear water-wave groups. Phys. Fluids 2002, 14, 3380-3390. [CrossRef]

28. Zhang, H.D.; Guedes Soares, C.; Onorato, M. Modelling of the spatial evolution of extreme laboratory wave heights with the nonlinear Schrödinger and Dysthe equations. Ocean Eng. 2014, 89, 1-9. [CrossRef]

29. Dyachenko, A.I.; Kachulin, D.I.; Zakharov, V.E. Super compact equation for water waves. J. Fluid Mech. 2017, 828, 661-679. [CrossRef]

30. Dyachenko, A.I.; Zakharov, V.E. Compact equation for gravity waves on deep water. JETP Lett. 2011, 93, 701-705. [CrossRef]

31. Dyachenko, A.I.; Zakharov, V.E. A dynamic equation for water waves in one horizontal dimension. Eur. J. Mech. B Fluids 2012, 32, 17-21. [CrossRef]

32. Dyachenko, A.I.; Zakharov, V.E. Spatial equation for water waves. JETP Lett. 2016, 103, 181-184. [CrossRef]

33. Coifman, R.; Meyer, Y. Nonlinear harmonic analysis and analytic dependence. Proc. Symp. Pure Math. 1985, 43, 71-78.

34. Craig, W.; Guyenne, P.; Hammack, J.; Henderson, D.; Sulem, C. Solitary water wave interactions. Phys. Fluids 2006, 18, 057106. [CrossRef]

35. Craig, W.; Guyenne, P.; Sulem, C. Water waves over a random bottom. J. Fluid Mech. 2009, 640, 79-107. [CrossRef]

36. Guyenne, P.; Nicholls, D.P. A high-order spectral method for nonlinear water waves over moving bottom topography. SIAM J. Sci. Comput. 2007, 30, 81-101. [CrossRef]

37. Berti, M.; Feola, R.; Pusateri, F. Birkhoff normal form for gravity water waves. Water Waves 2021, 3. [CrossRef]

38. Craig, W. Birkhoff normal forms for water waves. Contemp. Math. 1996, 200, 57-74.

39. Craig, W.; Guyenne, P.; Kalisch, H. Hamiltonian long-wave expansions for free surfaces and interfaces. Commun. Pure Appl. Math. 2005, 58, 1587-1641. [CrossRef]

40. Craig, W.; Sulem, C.; Sulem, P.-L. Nonlinear modulation of gravity waves: A rigorous approach. Nonlinearity 1992, 5, 497-522. [CrossRef]

41. Segur, H.; Henderson, D.; Carter, J.; Hammack, J.; Li, C.-M.; Pheiff, D.; Socha, K. Stabilizing the Benjamin-Feir instability. J. Fluid Mech. 2005, 539, 229-271. [CrossRef]

42. Keller, G.J. Experiments on nonlinear wave interaction. Private communication, 1982.

43. Su, M.Y. Evolution of groups of gravity waves with moderate to high steepness. Phys. Fluids 1982, 25, 2167-2174. [CrossRef]

44. Lake, B.M.; Yuen, H.C.; Ferguson, W.E. Envelope solitons and recurrence in nonlinear deep water waves: Theory and experiment. Rocky Mt. J. Math. 1978, 8, 105-116. [CrossRef]

45. Feir, J.E. Discussion: Some results from wave pulse experiments. Proc. R. Soc. Lond. A 1967, 299, 54-58.

46. Craig, W.; Schanz, U.; Sulem, C. The modulational regime of three-dimensional water waves and the Davey-Stewartson system. Ann. Inst. Henri Poincaré 1997, 14, 615-667. [CrossRef] 